\title{
Computed tomography assessment of the vascularization degree of hepatocellular carcinoma in the noncirrhotic liver
}

\author{
Ulyana N. Tumanova ${ }^{1,2}$, Grigoriy G. Karmazanovsky ${ }^{1}$, Alexander I. Shchyogolev ${ }^{2}$ \\ ${ }^{1}$ Department of Radiological Methods of Diagnosis and Treatment, A.V. Vishnevsky Institute of Surgery, Ministry of Health of Russia, \\ Moscow, Russia \\ ${ }^{2}$ Department of Pathology, Academician V.I. Kulakov Research Center of Obstetrics, Gynecology, and Perinatology, Ministry of Health of \\ Russia, Moscow, Russia
}

\section{Email address:}

u.n.tumanova@gmail.com (U. N. Tumanova),patan777@gmail.com (A. I. Shchyogolev)

\section{To cite this article:}

Ulyana N. Tumanova, Grigoriy G. Karmazanovsky, Alexander I. Shchyogolev. Computed Tomography Assessment of the Vascularization Degree of Hepatocellular Carcinoma in the Noncirrhotic Liver. American Journal of Clinical and Experimental Medicine.

Vol. 3, No. 1, 2015, pp. 24-31. doi: 10.11648/j.ajcem.20150301.13

\begin{abstract}
An important CT-characteristic at a diagnostic of liver tumors is the determination of its vascularity. To study the characteristics of blood tissue of hepatocellular carcinoma (HCC) with different degrees of differentiation by CT. The work is based on the clinical and morphological comparisons performed in 55 patients ( 34 men and 21 women aged 15-83 years) with hepatocellular carcinoma (HCC) without accompanying liver cirrhosis. In the preoperative stage all patients was carried spiral computed tomography (CT) with bolus contrast enhancement (on the four phases of the study). Was evaluated parameters of CT density and degree of nodes HCC vascularization. Morphologic study of histological preparations of operation material from 16 patients was diagnosed high-differentiated, 33 - moderately differentiated, and 6 - poorly differentiated HCC. At the immunohistochemical preparations with reaction to CD34 was determined by the number and the total cross-sectional area of vessels in sight of HCC tissue. The maximum values of CT density in the arterial phase were observed at the nodes moderately differentiated HCC. In the venous phase the greatest values of density as well as the venous increase was registered in highdifferentiated HCC. The most vascularised (hypervascular) tumor is high-differentiated HCC, and the least vascularized poorly differentiated HCC by CT. At morphometric study of the maximum values of the number and the total cross-sectional area of the blood vessels are installed in high-differentiated HCC tissues, the minimum - in the tissue of low-grade HCC. CT with bolus contrast enhancement allows studying the characteristics of blood supply of HCC varying degrees of differentiation. Determine the true measure of total CT-vascularization of the tumor tissue must be done with consideration of the increase concentration difference coefficient (ICDC).
\end{abstract}

Keywords: Hepatocellular Carcinoma, Histological Differentiation, Computed Tomography, Contrast Enhancement, Vascularity

\section{Introduction}

Hepatocellular carcinoma (HCC) is responsible for $85-90 \%$ of the total incidence of primary liver cancer. Every year in the world there are nearly 500,000 new cases and about the same number of patients die [1]. This considered, the incidence of HCC has tripled in the last twenty years, and the 5 -year survival rate is less than 5\% [2]. In Russia in 2013, the morbidity (ie, the number of patients per 100,000 people) of malignant tumors of the liver and intrahepatic bile duct was 4.7 [3].

The choice of therapeutic approach and life prognosis of patients with $\mathrm{HCC}$ are determined primarily by the volume and the functional state of the unaffected liver tissue, as well as the size, number and location of tumor nodes. The development of the majority of HCC nodes is observed in the setting of hepatic cirrhosis. However, the percentage of HCC developed in the absence of cirrhosis varies in different geographical areas from $7 \%$ to 54 [4].

Unfortunately, in the absence of liver cirrhosis, HCC is mainly diagnosed only at extensive (extreme) stages. For example, in Russia in 2013, the proportion of patients with neoplastic process stage III and IV, respectively, reaching $22.2 \%$ and $57.1 \%$. Consequently, the mortality rates of HCC patients within a year from the time of diagnosis have the 
highest value among all the tumors, accounting for $69.9 \%$ [3].

The leading role in the diagnostics and subsequent treatment, as well as disease prognosis reasonably belongs to radiological methods, particularly computed tomography, due to their non-invasive character, highly informative value and speed $[5,6]$.

However, an important role in the development and progression of HCC is given to vascularization processes, that is, the formation of new blood vessels and blood supply characteristics, and the potential methods of treatment include the development of anti-angiogenic drugs. Therefore, the identification of HCC development, progression, and correction processes associated with angiogenesis is an important task [7, 8]. We have previously demonstrated that the morphological characteristics of HCC vascularization depend on the degree o histological differentiation [9].

In this paper, we used spiral CT to study the peculiarities of HCC tissue blood supply depending on the degree of histological differentiation in the noncirrhotic liver.

\section{Material and Methods}

\subsection{Patients}

The work is based on the clinical and morphological comparisons performed in 55 patients with hepatocellular carcinoma (HCC) at A. V. Vishnevsky Institute of Surgery of the Ministry of Health of the Russian Federation (Moscow, Russia) in 2007-2009 (Table 1).

Table 1. Patient Characteristics

\begin{tabular}{ll}
\hline & No. of Patients \\
\hline men & 34 \\
women & 21 \\
age median (oscillations), year & $55,9(15-83)$ \\
localization (right lobe / left lobe) & $41 / 23$ \\
HCC & \\
well differentiated & 16 \\
moderately differentiated & 33 \\
poorly differentiated & 6 \\
\hline
\end{tabular}

\subsection{Computed Tomography}

In the preoperative stage, all the patients underwent spiral computed tomography (CT) with bolus contrast enhancement with Philips Brilliance 64 CT. Both the contrast medium and sodium chloride injections were carried out using the automatic double-head injector OptiVantage DH (Mallinckrodt; Inc) at a rate of $4 \mathrm{ml}$ per sec. Bolus tracking software package was used to start the scanning. Four phases were analyzed: native, arterial, venous, and delayed. For arterial and venous phases, scanning was started 10 and 30 seconds after reaching the predetermined threshold aorta opacification respectively. The delayed phase was performed in all patients after 4-5 min after the administration of contrast medium. The obtained CT scans were used to determine the location, size, and boundaries of the tumor, as well as the densitometric parameters of the tumor tissue surrounding liver parenchyma, aorta in the celiac artery and the portal vein in its bifurcation.

On the basis of the densitometric indices obtained in different phases of scanning, calculated values of arterial (AI) and venous (VI) increase of the tumor density were calculated. The arterial increase was calculated as the difference in the CT tumor density values in the arterial and the native phase. The venous increase was calculated as the difference between the CT tumor densities in the arterial and venous phases, taking into account the increase concentration difference coefficient (ICDC) in the aorta and the portal vein. Negative values of VI indicated the absence of additional blood flow and thus the presence of contrast medium outflow from the tumor in the venous phase. In these cases, the difference in the CT densities in the arterial and the venous phase is regarded as zero. Using ICDC allows to mathematically neutralize the difference in the concentrations of the contrast medium in affering blood vessels (aorta and portal vein) and thus to determine the true value of CT vascularization index of liver and tumors. It is calculated as the ratio of the increase in the density of the aorta in the arterial phase (in relation to the native) to the values of density increase in portal vein in a venous phase (in relation to the native).

Index of $\mathrm{CT}$ vascularization degree corresponding to the sum of the arterial (AI) and venous (VI) increase values reflects the level of accumulation of contrast medium entering through hepatic artery and portal vein, and hence gives an idea of the total quantity of the inflowing blood. Index of CT vascularization calculated by the formula: (AI) + (VI x ICDC).

\subsection{Morphological Analysis}

The surgical material obtained after resection was subjected to a comprehensive macro- and microscopic examination. A histological examination was performed on paraffin sections stained with hematoxylin and eosin. The degree of histological differentiation was determined according to the recommendations of the WHO International histological classification of tumors of the liver [10]. 16 patients were diagnosed with well differentiated (WD), 33 with moderately differentiated (MD) and 6 with the poorly differentiated (PD) variant of HCC.

Detection of blood vessel tissue specimens was performed by immunohistochemistry using ready-to-use mouse monoclonal antibodies to CD34 (QBEnd/10 clone) and Spring Bioscience system for polymer system production detection. The preliminary antigen unmasking was performed by boiling samples in solution with citrate buffer, $\mathrm{pH} 6.0$. Block endogenous peroxidase was performed by treatment of the sections with $0.3 \%$ hydrogen peroxide solution for 15 minutes. As the background stain, hematoxylin was used.

The so-called hot spots in immunohistochemical preparations were detected, where the number and the total cross-sectional area of the blood vessels were determined using the Axio Imager M1-based image analysis system with the help of AxioVision software (Carl Zeiss, Germany). 


\subsection{Statistical Analysis}

Statistical analysis of the results was performed using Statistica 8.0 Portable. The mean value (M) and standard deviation (sd) were calculated. Statistical significance was considered at $\mathrm{p}$ less than 0.05 .

\section{Results}

\subsection{Computed Tomographic Characteristics of HCC of Various Degrees of Differentiation}

During the computed tomography study, the density of HCC, as well as the blood in blood vessels (aorta and portal vein) supplying the liver depended on the phase of the study (fig.1-3). In the native phase, the densities of WD HCC were slightly higher than the corresponding values of MD and PD HCC ( $>>0.05)$ (Table. 2). After the injection of the contrast medium, an increase of density values was detected in all the groups studied (fig.4). The most pronounced rise, and thus an arterial increase, was registered with MD HCC. As a result, the maximum value of the $\mathrm{CT}$ density in the arterial phase was also observed in MD HCC. In the venous phase, the highest values of density as well as a venous increase were registered in WD HCC.

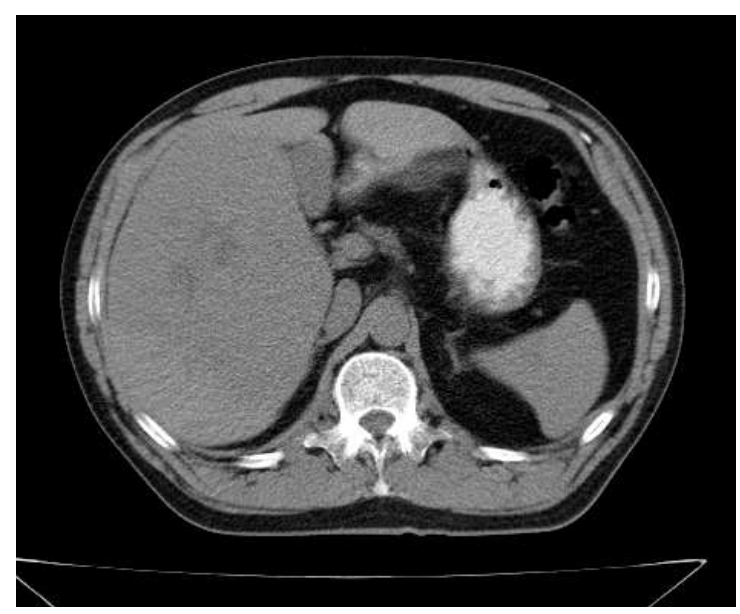

a

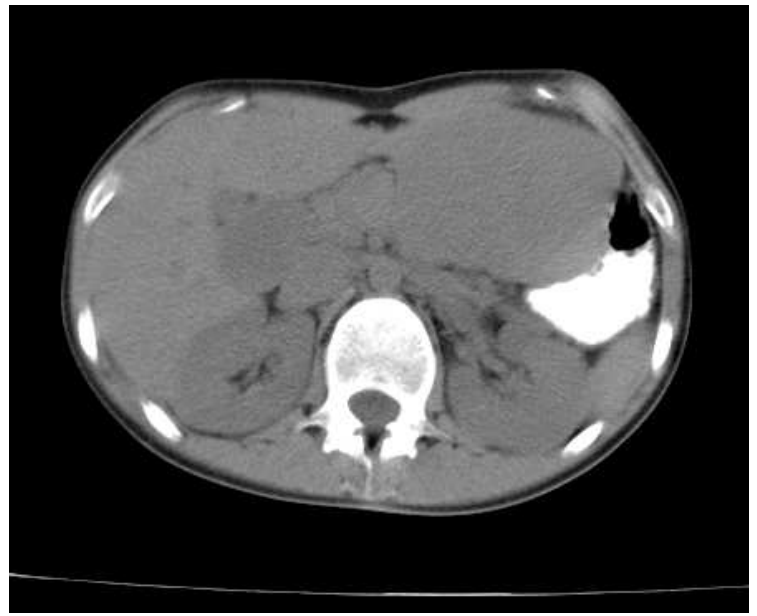

b

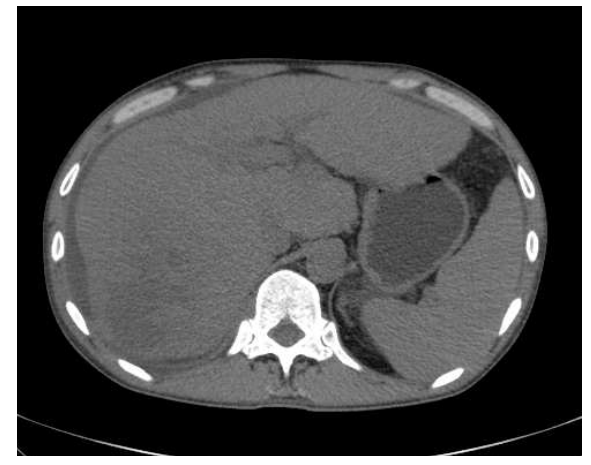

c

Figure 1. Computed tomographic characteristics of WD (a), MD (b), PD (c) HCC at native phase.

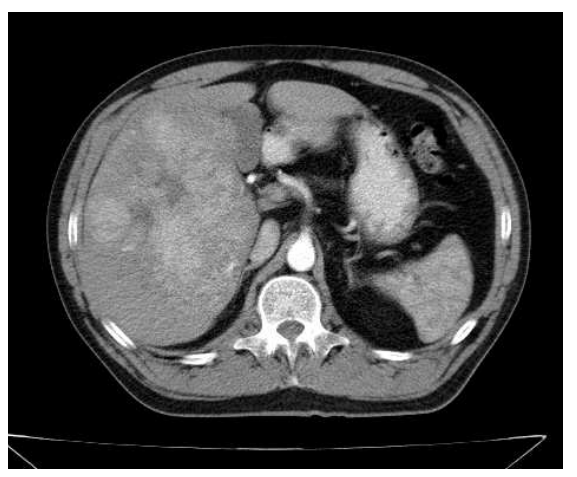

a

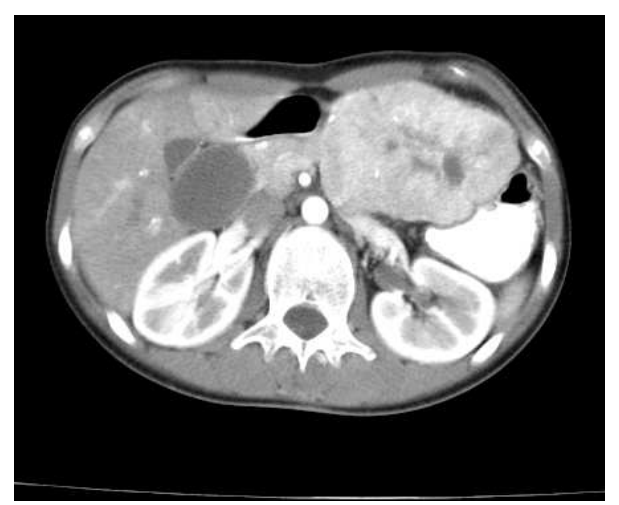

b

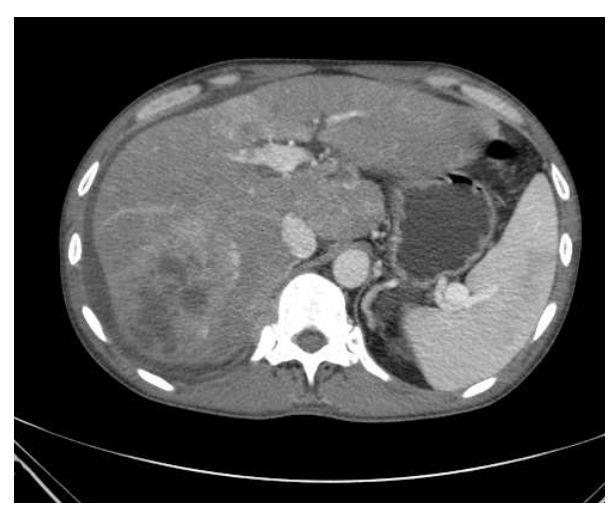

$\mathrm{c}$

Figure 2. Computed tomographic characteristics of WD (a), MD (b), PD (c) HCC at arterial phase. 


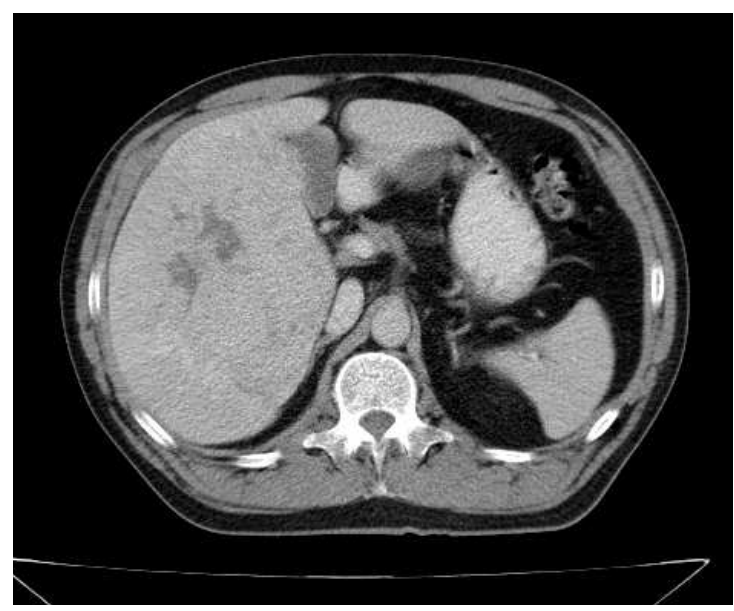

a

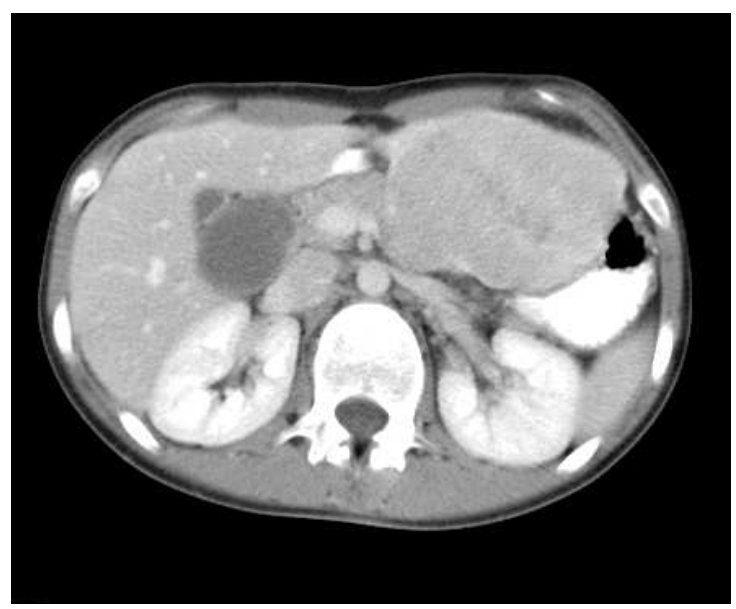

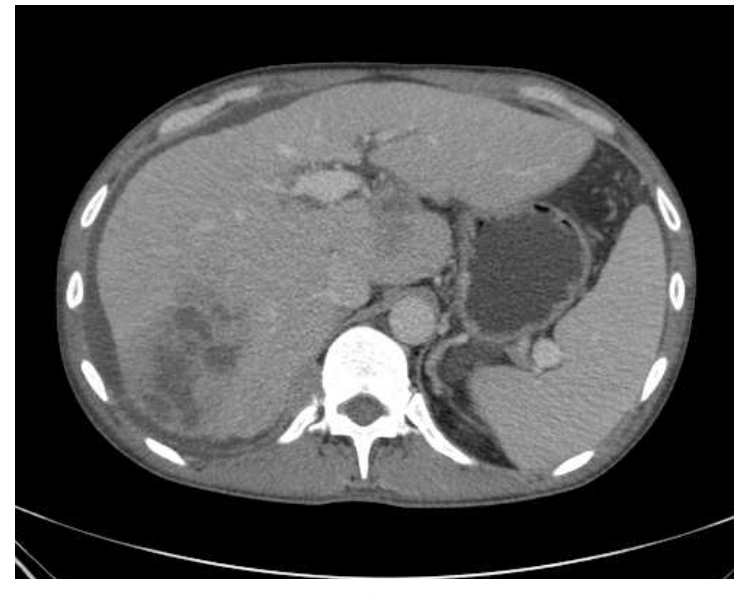

c

Figure 3. Computed tomographic characteristics of WD (a), MD (b), $P D(c)$ HCC at venous phase.

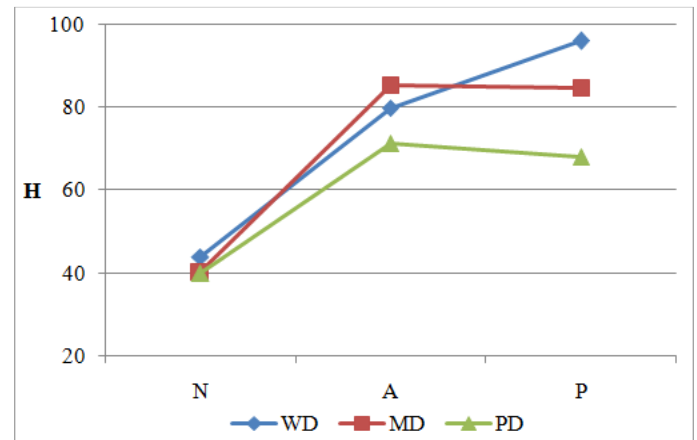

Figure 4. Dynamics of change of indicators CT-density HCC varying degrees of histological differentiation

Table 2. CT-density of HCC tissue varying degrees of differentiation in different phases of the study $(M \pm s d$, un.H)

\begin{tabular}{llll}
\hline phase of the study & WD & MD & PD \\
\hline native phase & $43,7 \pm 11,6$ & $40,1 \pm 7,1$ & $39,8 \pm 11,4$ \\
arterial phase & $79,7 \pm 19,6$ & $85,5 \pm 22,7$ & $71,2 \pm 14^{\mathrm{b}}$ \\
density increase in the arterial phase compared to native (arterial increase) & $36,0 \pm 15,6$ & $45,4 \pm 22,9$ & $31,4 \pm 13,0^{\mathrm{b}}$ \\
venous phase & $96,0 \pm 16,7$ & $84,8 \pm 22,5$ & $68,0 \pm 14,0^{\mathrm{a}} \mathrm{b}$ \\
density increase in the venous phase compared to arterial & $16,3 \pm 7,9$ & $-0,7 \pm 22,2^{\mathrm{a}}$ & $-3,2 \pm 11,3^{\mathrm{a}}$ \\
venous increase & $16,3 \pm 7,9$ & $9,2 \pm 11,5^{\mathrm{a}}$ & $3,2 \pm 3,8^{\text {a }}$ \\
\hline
\end{tabular}

a - $\mathrm{p}<0,05$ compared to WD HCC, ${ }^{\text {b }}$ - $p<0,05$ compared to MD HCC

Table 3. $C T$ density in the lumen of the aorta $(A)$ and the portal vein $(P V)$ at CT-study HCC of different degree of differentiation (M $\pm s d$, un.H)

\begin{tabular}{lllll}
\hline phase of the study & vessel & WD & MD & PD \\
\hline \multirow{2}{*}{ native phase } & A & $37,9 \pm 7$ & $39,8 \pm 5,5$ & $33,8 \pm 5,3$ \\
& PV & $42,0 \pm 9,3$ & $37,0 \pm 6,1$ & $40,2 \pm 21,7$ \\
arterial phase & A & $268,3 \pm 52,3$ & $267,2 \pm 76,8$ & $222,8 \pm 59,6$ \\
& PV & $87,1 \pm 24,7$ & $95,3 \pm 44,5$ & $107,7 \pm 26,2$ \\
venous phase & A & $111,1 \pm 12,4$ & $120,4 \pm 16,0$ & $116,8 \pm 12,2$ \\
density increase in the arterial phase compared to native & PV & $127,6 \pm 15,7$ & $123,6 \pm 20,6$ & $114,7 \pm 18,2$ \\
density increase in the venous phase compared to native & A & $230,4 \pm 52,7$ & $227,4 \pm 74,9$ & $189,0 \pm 57,3$ a b \\
increase concentration difference coefficient (ICDC) & PV & $85,6 \pm 12,5$ & $86,6 \pm 19,1$ & $74,5 \pm 22,5$ \\
\hline
\end{tabular}

Computed tomographic density values of the portal vein and aorta are shown in Table. 3. The arterial phase was characterized by a more pronounced increase in density in the aorta compared with the portal vein. The venous phase was, in contrast, characterized by a decrease in arterial CT density in the aorta, and vice versa, an increase in density in the portal vein. The calculated ICDC values did not depend on the degree of histological differentiation and amounted to 2.7 (table 3 ). This measure should be considered separately for each case to take into account the individual differences 
in dynamics of contrast enhancement, depending on heart rate, body weight, blood volume of the patient, etc. The individual variation rate was from 0.98 to 4.3 .

The calculated CT measure of HCC vascularization had maximum values at WD HCC exceeding the M D and PD HCC values by $14,0 \%$ and $100,0 \%(p<0,05)$, respectively (fig. 5).

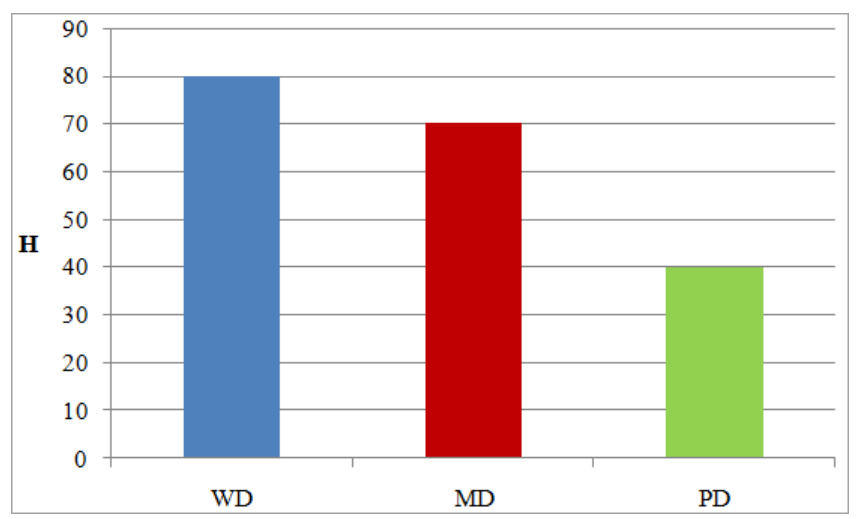

Figure 5. Index of CT vascularization HCC varying degrees of histological differentiation

\subsection{Morphological Indicators of HCC Tissue} Vascularization of Various Degrees of Differentiation

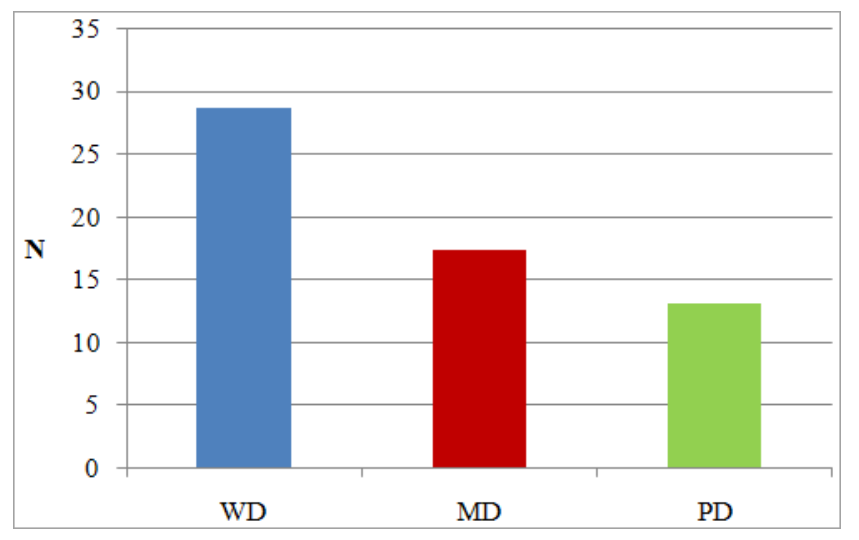

a

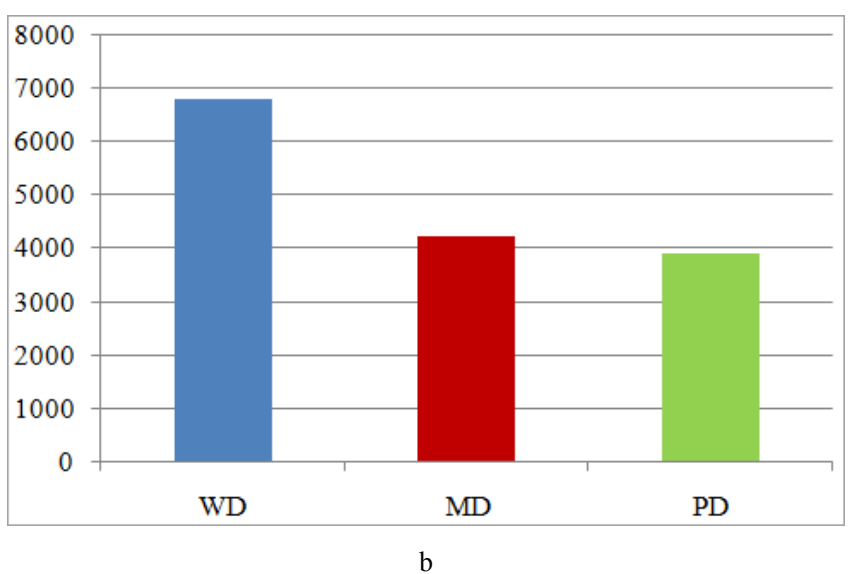

Figure 6. The amount (a) and the total cross-sectional area $\left(\mu^{2}\right)$ (b) of vessels in the tissue of HCC varying degrees of histological differentiation
A morphometric analysis of immunohistochemical preparations has showed that the maximum quantity and the total cross-sectional area of blood vessels was observed in WD HCC tissue (Table. 4). The number of vessels in WD HCC tissue exceeds the similar values in MD and PD HCC by $65.9 \%$ and $119.1 \%$, respectively $(\mathrm{p}<0.05)$. The total crosssectional area of WD HCC vessels exceeds the similar values in $\mathrm{MD}$ and $\mathrm{PD} \mathrm{HCC}$ by $60.3 \%$ and $73.6 \%$, respectively $(\mathrm{p}<0.05)$ (fig. 6).

\section{Discussion}

Hepatocellular carcinoma (HCC) is one of the leaders in terms of tumor morbidity and mortality [11]. This considered, the incidence of HCC has tripled in the last twenty years, and the 5-year survival rate is less than 5\% [2].

Since HCC refers to tumors with rapid growth and a pessimistic prognosis, including the cases with an absence of cirrhosis, a possibility for a clear unambiguous diagnostics of HCC becomes particularly important. The main methods of non-invasive diagnostics and designation of the subsequent treatment and prognosis of $\mathrm{HCC}$ are considered to be radiological methods.

The use of CT with bolus contrast enhancement allows to obtain a tomographic image in different phases (arterial, venous, delayed), and thus to compare the characteristics of tumor, blood vessels and organ parenchyma opacification, with their image in the non-contrast phase of the study. The use of spiral CT with bolus contrast enhancement allows to compare densitometric indices of various organs, as well as the tumor and the surrounding tissue (parenchyma) in the affected organ in the degree of contrast medium accumulation. Since the contrast medium flows through the blood vessels, the organs and tumors with a higher CT density in the arterial phase of the study are considered hyperdense (or hypervascular) and those with a lower density are considered hypodense (or hypovascular) [12].

For example, the spleen is a more vascularized organ compared with the liver [13], the mucous membrane of the stomach and intestines is hypervascular compared with the muscle layer [14]

Hypervascular liver tumors include cavernous hemangioma, focal nodular hyperplasia, metastatic neuroendocrine carcinoma, breast and thyroid, renal cell carcinoma, melanoma [5].

However, describing CT signs and determining the true vascular component saturation of the focal lesions of the liver, such as HCC, it is important to take into account the peculiarities of liver blood supply. Under normal conditions, about $75-80 \%$ of the blood flows through the portal vein, the remaining $20-25 \%$ through the hepatic artery. Thereby the maximum accumulation of the contrast medium by liver parenchyma is observed in the portal (venous) phase of the CT study.

Most tumors of the liver have almost exclusively arterial blood supply (via the hepatic artery) [15]. For example, most of the blood supply to colon cancer metastases is $95 \%$ arterial 
[16]. However, some tumors of the liver have a partially preserved portal inflow, the extent of which also needs to be adequately taken into account in determining the total node vascularization.

However, most researchers point out that the dynamics of densitometric indices chane and thus the tumor type (hyper-, hypo- or isodense) in each phase of the scan depends on the size and the condition of the liver tissue (especially the presence of cirrhosis). The observations we studied had no evidence of cirrhosis.

According to the literature, about a third observations of the smallest (less than $2 \mathrm{~cm}$ ) $\mathrm{HCC}$ in the native phase of CT studies appears hypodense [17, 18], and larger sizes tumors appear hypersense in relation to the surrounding liver tissue. In the arterial phase tumors usually tend to become hyperdense[19].

It is the identification of arterial hypervascular nodes larger than $2 \mathrm{~cm}$ in liver cirrhosis with $\mathrm{CT}$ and/or MRI is, in accordance with the recommendations of the American Association for the Study of Liver Diseases, the basic diagnostic criterion for HCC [20].

Describing the features of the blood supply to tumors of the liver, it should be noted that intranode hemodynamics and blood inflow change along with the increasing degree of tissue and cell irregularities [21]. Hepatocarcinogenesis is a multistep process of hyperplastic nodes canceration into an early cancer and ultimately into an expressed carcinoma. In the initial phase of carcinogenesis (in hyperplastic node), hemodynamic parameters indicate a predominantly portal perfusion (through the portal vein) and arterial hypovascularity. Then a decrease in both venous and arterial blood inflow is detected. Later, there is an increase in blood flow within the node, resulting in node first becoming isovascular, and ultimately hypervascular.

Development of HCC is characterized not only by increased cell irregularities with a reduction in the degree of histological differentiation, but also angioarchitectonic changes in the tumor tissue [22]. According to [23], the progression of HCC is accompanied by a decrease in the number of portal tracts and the formation of so-called unpaired arteries, which accordingly reduces the portal inflow of blood and an increase in the arterial inflow. Subsequently, in the tumor tissue sinusoid spaces exposed to capillarization are formed [24]. Perhaps that is why the greatest indicators of CT density of tumor tissue are registered and in the arterial phase for MD and in the venous phase for WD HCC. According to our research (Table. 2), the decrease in the degree of histological differentiation of HCC node is associated with an increase in the proportion of blood flowing to the hepatic artery. In WD HCC observations proportion of arterial blood is $45,0 \%$, in the MD HCC - 64,7\% and PD HCC - 78,5\%. Simultaneously there is a decline in the proportion of blood flowing through the portal vein. For WD HCC nodes portal inflow is $55,0 \%$, MD HCC $-35,3 \%$ and PD HCC - 21,5\%.

Thus, the CT examination with contrast enhancement allows to estimate the ratio of the degree of tumor vascularization in relation to parenchymal organ vascularization in vessels engaged in blood flow to the normal and pathological tissues. The larger vessels supply blood to the tumor, the more intensive the accumulation of contrast medium is in a particular phase of the scan. Therefore, it is advisable to assess vascularization density according to the tumor growth in different phases of the study. This considered, hyper- and hypovascular tumors are characterized by a certain type of staining, including in MRI studies.

In hypervascular tumors, an increased contrast enhancement is found compared to the surrounding parenchyma in the arterial phase, so that they usually look like plots of transient hyperdensity. For such tumors, an early (the first $25 \mathrm{~s}$ ) opacification with uneven and inhomogeneous accumulation of contrast medium with late sac enhancement [25].

In hypovascular tumors, perfusion less than the surrounding parenchyma, so they become more distinct in the phase of maximum contrast enhancement of the organ parenchyma. For hypovascular carcinomas, negative staining in the portal phase and positive sac opacification is typical, and only in interstitial phase a slight opacification of the tumor occurs due to diffusion of the contrast medium.

To be fair, we must take into account the difference in the initial values of structures and organs density. Therefore, it is in our opinion more correctly to evaluate the vascularity by the density growth of the tumor and unaffected tissue, thereby taking into account the initial values.

Indeed, quite often on the visual picture, as well as densitometric parameters, hypervascular tumors in the arterial phase of scanning have higher densities than the surrounding parenchyma. However, due to the summation of the initial densities of the tumor and organ parenchyma with the values of their density increase at opacification, the tumor may have lower values in relation to the organ parenchyma, being a hypervascular tumor. This happens due to the fact that even with a higher gradient of growth in this opacification phase, the node does not compensate for initial difference in densities with organ parenchyma, and thereby the tumor is visually defined as hypodense area. Often this can be observed with concomitant lesions of the liver parenchyma, accompanied by changes in the initial density (e.g. in the setting of hepatitis or cirrhosis).

In view of the above, $f$ correct judgment of hyper- or hypovascularity of the tumor is only possible by the ratio of the tumor and the surrounding organ parenchyma density increase gradient in the arterial or venous phase, and not by densitometric difference or visual picture.

Due to the peculiarities of the liver and pathological formations structure described above, the accumulation of contrast medium in the parenchyma is carried out in two phases: the arterial and the venous. That is why when studying the liver, we must take into account the parameters of these two phases, as well as the parameters of maximum accumulation of contrast medium. Thus, the best method of determining the type of vascular tumors of the liver, in our opinion is to calculate the difference between the maximum increase in tumor density and the maximum increase in the liver tissue, regardless of the phase in which it happened. 
Evaluating the results of our morphometric analysis of immunohistochemical drugs, it is important to note that in the literature there is still ambiguity in the meaning of the term "degree of vascularization" and consequently, its definition (calculation). The term 'vascularization' is mainly morphological. A number of authors define it as the microvessel count in the field (in particular, $0.72 \mathrm{~mm}^{2}$ given a magnification of 20) [26]. Other morphologists calculate it as the relative (total) vascularity jf the cross-sectional area of the blood vessels in the microscope field [27]. We agree with the last point of view, since the total cross-sectional area of vessels depends on their number and diameter.

CD34 antibodies are most commonly used to assess liver oncogenesis in tumors. Despite the fact that CD34 is a panendothelial marker and not a specific neoangiogenesis marker, this reaction is used for the differential diagnostics of tumors and prognosis of liver diseases [28].

According to the literature [29], the microvessel density detected using CD34 antibodies in early HCC tissue (0.043 \pm $0.027)$ is higher than those in regenerative $(0.007 \pm 0.006)$ and dysplasia $(0.011 \pm 0.009)$ nodules of hepatocytes. Similar results were obtained by I. Chebib et al. in 2007 [30]. The above changes confirm the data above about the change of immunophenotype of endothelial cells during hepatocarcinogenesis. According to our previous studies, the degree of HCC vascularization depends on the degree of its histological differentiation: with a decrease in the degree of HCC differentiation, there is a progressive decline in the number of blood vessels [9].

By means of multiple regression analysis, it is revealed that of all the studied parameters of HCC only tumor size correlated with the density of CD34 positive performance vessels. Thus, the mean density values in nodes with a diameter less than $5 \mathrm{~cm}\left(316 / 0.74 \mathrm{~mm}^{2}\right)$ was more than 2 times higher than those $\left(146 / 0.74 \mathrm{~mm}^{2}\right)$ in tumors larger than $5 \mathrm{~cm}(\mathrm{p}<0.001)$ [31].

It should also be noted that the maximum density values are observed in the peripheral areas of HCC [31]. Density values in HCC tissue that developed in the setting of of hepatitis $\mathrm{C}(54 \pm 13.8)$ are higher than the corresponding values for HCC caused by to hepatitis $\mathrm{B}(38 \pm 8.9)$ [32]. In addition, children with HCC have a significantly higher microvessel density compared with adult patients (36.94 and 26.52, p <0.05), but no significant difference with other childhood malignancies [33].

Our morphometric study established differences in the number and the total cross-sectional area in HCC tissue vessels of various degrees of histological differentiation. A reduction in HCC histological differentiation (with a respective increase in malignance) is accompanied by a decrease in the number and total area of the vessels. In our opinion, to assess the degree of vascularization of HCC on immunohistochemical preparations advisable to use both indicators: the number of microvessels and total (specific) area of the cross section. The observed changes are consistent with the literature on the lower values of the density of intratumoral vessels in larger tumors, and at later stages of tumor development [34]. Indeed, pronounced angiogenesis is characteristic of early stages of tumor development, it is later reduced. During tumor development and increase in size, the metabolism of tumor cells changes to anaerobic [35]. These data indicate attenuation of neoangiogenesis during HCC progression and growth, which, in our opinion, should be considered in the development of anti-angiogenic drugs and their prescription to patients suffering from HCC.

\section{Conclusion}

In summary, using spiral CT along with with bolus contrast enhancement allows to explore the peculiarities of blood supply in HCC of various stages of differentiation. The most vascular (hypervascular) tumor, according to by CT and the results of immunohistochemical studies is the highly differentiated, and the least vascular is the low-grade variant of HCC. Determination of the true CT indicator of tumor tissue vascularization in the liver should be carried out taking into account the difference coefficient of inflow concentrations. We believe that only the quantitative assessment of the difference in contrast concentrations of inflow systems leads to an objectively true image analysis in different phases of CT examinations and thus to an accurate conclusion about the peculiarities of tumor blood supply. The presented method can be recommended to assess the degree of vascularization of $\mathrm{HCC}$ in patients with chemotherapy. In this regard, RECIST tumor evaluation tumors can become more objective and allow to continue chemotherapy even in those cases, where according to the visual assessment of $\mathrm{CT}$ images a lack of response to therapy was detected.

\section{References}

[1] K. A. McGlynn, L. Tsao, A.W. Hsing, S.S. Devesa, and J.F. Fraumeni Jr, International trends and patterns of primary liver cancer, Int J Cancer. 2001; 94(2): 290-6.

[2] H. B. El-Serag, and K. L. Rudolph, Hepatocellular carcinoma: epidemiology and molecular carcinogenesis, Gastroenterology. 2007; 132(7): 2557-6.

[3] State of cancer care in Russia in 2013 / Eds. A. D. Caprin, V. V. Starinskiy, and G.V. Petrova. M.: MNIOI them. P.A. Herzen of Russian Ministry of Health, 2014. p.235 [in Russian]

[4] F. Trevisani, M. Frigerio, V. Santi, A. Grignaschi, and M. Bernardi, Hepatocellular carcinoma in non-cirrhotic liver: a reappraisal, Dig Liver Dis. 2010; 42(5):341-7.

[5] A. C. Silva, J. M Evans, A. E. McCullough, M. A. Jatoi, H. E. Vargas and A.K. Hara, MR Imaging of hypervascular liver masses: A review of current techniques, Radiographics. 2009; 29(2):385-402.

[6] S. Gaddikeri, M. F. McNeeley, C. L. Wang, P. Bhargava, M. K. Dighe, M. M. Yeh, T.J. Dubinsky, O. Kolokythas and N. Lalwani, Hepatocellular carcinoma in the noncirrhotic live, Am J Roentgenol. 2014; 203(1): W34-47. 
[7] T. K. Lee, R. T. Poon, A. P. Yuen, M. T. Ling, X. H. Wang, Y. C. Wong, X. Y. Guan, K. Man, Z. Y. Tang, and S. T. Fan, Regulation of angiogenesis by Id-1 through hypoxia-inducible factor-1alpha-mediated vascular endothelial growth factor upregulation in hepatocellular carcinoma, Clin Cancer Res. 2006;12(23): 6910-19.

[8] A. X. Zhu, D. G. Duda, D. V. Sahani, and R. K. Jain, HCC and angiogenesis: possible targets and future directions, Nat Rev Clin Oncol. 2011;8(5): 292-301.

[9] A. I. Shchyogolev, E. A. Dubova, and U. N. Tumanova, Vascularization of hepatocellular carcinoma tissue depends on its differentiation degree, Bull of Experimental Biology and Medicine. 2012; 153(4):490-4.

[10] WHO classification of tumours of the digestive system / Eds. N. D. Theise, M. P. Curado, S. Franceschi et al. - Lyon: IARC, 2010. P.205-16.

[11] H. B. El-Serag, Hepatocellular carcinoma: recent trend in the United States, Gastroenterology. 2004; 127(5, Suppl 1): S27S34.

[12] A. Qayyum, A. Graser, A. Westphalen, R. B. Merriman, L. D. Ferrell, B. M. Yeh, and F. V. Coakley, CT of benign hyper vascular liver nodules in autoimmune hepatitis, Am J Roentgenol. 2004; 183(6):1573-6.

[13] M. Hofer, CT teaching manual. Georg Thieme Verlag, 2000.$176 \mathrm{p}$.

[14] N. K. Lee, S. Kim, G. H. Kim, T. Y. Jeon, D. H. Kim, H. J. Jang, and do Y Park, Hypervascular subepithelial gastrointestinal masses: CT-pathologic correlation, Radiographics. 2010; 30(7):1915-34.

[15] O. Matsui, M. Kadoya, T. Kameyama, J. Yoshikawa, T. Takashima, Y. Nakanuma, M. Unoura, K. Kobayashi, R. Izumi, M. Ida, et al. Benign and malignant nodules in cirrhotic livers: distinction based on blood supply, Radiology. 1991;178(2):4937.

[16] A. D. Cohen, and N. E. Kemeny, An update on hepatic arterial infusion chemotherapy for colorectal cancer, The Oncologist. 2003; 8(6):553-6.

[17] M. Kudo, Imaging diagnosis of hepatocellular carcinoma and premalignant/borderline lesions, Semin Liver Dis. 1999; 19(3):297-309.

[18] L. Bolondi, S. Gaiani, N. Celli, R. Golfieri, W.F. Grigioni, S. Leoni, A.M. Venturi, and F. Piscaglia, Characterization of small nodules in cirrhosis by assessment of vascularity: the problem of hypovascular hepatocellular carcinoma, Hepatology. 2005; 42(1):27-34.

[19] E. K. Outwater, Imaging of the liver for hepatocellular cancer, Cancer Control. 2010; 17(2):72-82.

[20] J. Bruix, and M. Sherman, Practice Guidelines Committee, American Association for the Study of Liver Diseases. Management of hepatocellular carcinoma, Hepatology. 2005; 42(5):1208-36.

[21] M. Kudo, Morphological diagnosis of hepatocellular carcinoma: special emphasis on intranodular hemodynamic imaging, Hepatogastroenterology. 1998;45(suppl 3):1226-31.

[22] M. Kojiro, Pathology of hepatocellular carcinoma.
Massachusetts: Blackwell Publishing, 2006, p.63-76.

[23] Y. Nakashima, O. Nakashima, C.C. Hsia, M. Kojiro, and E. Tabor, Vascularization of small hepatocellular carcinomas: correlation with differentiation, Liver.1999; 19(1):12-8.

[24] M. Kin, T. Torimura, T. Ueno, S. Inuzuka, and K. Tanikawa, Sinusoidal capillarization in small hepatocellular carcinoma, Pathol Int. 1994;44(10-11):771-8.

[25] A. Kamaya, K. E. Maturen, G. A. Tye, Y. I. Liu, N. N. Parti, and T. S. Desser, Hypervascular liver lesions, Semin Ultrasound CT MR. 2009;30(5):387-407.

[26] C-N. Chen, Y-M. Cheng, J-T. Liang, P. H. Lee, F. J. Hsieh, R. H. Yuan, S. M. Wang, M. F. Chang, and K. J. Chang, Color doppler vascularity index can predict distant metastasis and survival in colon cancer patients, Cancer Res. 2000;60(11):2892-7.

[27] W. Zeng, A. S. H. Gouw, M. C. van den Heuvel, J. Molema, S. Poppema, E. J. van der Jagt, and K. P. de Jong, Hepatocellular carcinomas in cirrhotic and noncirrhotic human livers share angiogenic characteristics, Ann Surg Oncol. 2010; 17(6):1564-71.

[28] Y. Yao, Y. Pan, J. Chen, X. Sun, Y. Qiu, and Y. Ding, Endoglin (CD105) expression in angiogenesis of primary hepatocellular carcinomas: analysis using tissue microarrays and comparisons with CD34 and VEGF, Ann Clin Lab Sci. 2007;37(1):39-48.

[29] J. P. Paschoal, V. Bernardo, N. H. S. Canedo, O. D. Ribeiro, A. Caroli-Bottino, and V. L. Pannain, Microvascular density of regenerative nodule to small hepatocellular carcinoma by automated analysis using $\mathrm{CD} 105$ and $\mathrm{CD} 34$ immunoexpression, BMC Cancer. 2014; 14:72.

[30] I. Chebib, M. T. Shabani-Rad, M. S. Chow, Y. Zhang, and Z. H. Gao, Microvessel density and clinicopathologic characteristics in hepatocellular carcinoma with and without cirrhosis, Biomarker Insights. 2007; 14(2):59-68.

[31] R. T. Poon, I. O. Ng, C. Lau, W. C. Yu, Z. F. Yang, S. T. Fan, and J. Wong, Tumor microvessel density as a predictor of recurrence after resection of hepatocellular carcinoma: a prospective study, J Clin Oncol. 2002; 20(7):1775-85.

[32] L. Messerini, L. Novelli, and C. E. Comin, Microvessel density and clinicopathological characteristics in hepatitis $\mathrm{C}$ virus and hepatitis B virus related hepatocellular carcinoma, $\mathrm{J}$ Clin Pathol. 2004; 57(8):867-71.

[33] X. Y. Sun, Z. D. Wu, X. F. Liao, and J. Y. Yuan, Tumor angiogenesis and its clinical significance in pediatric malignant liver tumor, World J Gastroenterol. 2005;11(5):741-3.

[34] O. N. El-Assal, A. Yamanoi, Y. Soda, M. Yamaguchi, M. Igarashi, A. Yamamoto, T. Nabika, and N. Nagasue, Clinical significance of microvessel density and vascular endothelial growth factor expression in hepatocellular carcinoma and surrounding liver: possible involvement of vascular endothelial growth factor in the angiogenesis of cirrhotic liver, Hepatology. 1998; 27(6):1554-62.

[35] L. Hlatky, P. Hahnfeldt, and J. Folkman, Clinical Application of Antiangiogenic Therapy: Microvessel Density, What It Does and Doesn't Tell Us, J Natl Cancer Inst. 2002;94(12):883-93. 